\title{
LAS FALLAS EN LA INGENIERÍA DE REQUISITOS
}

\author{
Lindsey Westfall \\ University of Texas \\ LWestfall@utexas.edu
}

(Tipo de artículo: INVESTIGACIÓN. Recibido el 15/08/2011. Aprobado el 20/11/2011)

\begin{abstract}
RESUMEN
Recientemente, la Ingeniería de Requisitos ha cobrado una importancia cada vez mayor en la academia y en la industria, debido a que se espera que los productos software de este siglo proporcionen funciones cada vez más centradas en el usuario y de mayor calidad y seguridad. Por lo tanto, es importante comprender las situaciones en las que la práctica actual de la Ingeniería de Requisitos -IR- no funciona adecuadamente. Este documento presenta los resultados de una investigación acerca de las prácticas de IR. Se realizó a través de encuesta web y se focalizó en las técnicas de elicitación de requisitos y en las técnicas de representación de requisitos que los encuestados utilizan. A pesar de que los resultados son el reporte acerca del estado del arte de la Ingeniería de Requisitos para la población que respondió la encuesta, es probable que también refleje el estado del arte de la IR en todo el mundo.
\end{abstract}

\section{Palabras clave}

Ingeniería de Requisitos, Elicitar, Técnicas de Elicitación, Técnicas de Representación.

\section{THE FAILURES OF THE REQUIREMENTS ENGINEERING}

\begin{abstract}
Recently, Requirements Engineering has gained increasing importance in academy and industry, because it is expected that software products of this century will provide functions focused on the user and exhibiting higher quality and safety. Therefore, it is important to understand the situations in which the current practice of the Requirements Engineering -RE- does not work properly. This document presents the results of a research about RE practices. The research was conducted through a web survey and was focused on the techniques of requirements elicitation and representation techniques of requirements used by those polled. Although the main result is a report on the state of the art of Requirements Engineering for those polled, it is also probable that can be reflected the RE's state of the art worldwide.
\end{abstract}

\section{Keywords}

Requirements engineering, to elicit, elicitation techniques, representation techniques.

\section{L'ÉCHEC DE LA GÉNIE DES EXIGENCES}

\section{RESUME}

Récemment, la Génie des Exigences a repris une importance plus grande dans le milieu académique et industriel, parce qu'on espère que les produits logiciels du siècle actuel fournissent des fonctions de plus en plus dirigés vers l'utilisateur et d'une qualité et sécurité plus grandes. Par conséquent, est important de comprendre les situations dans lesquelles la pratique actuelle de la Génie des Exigences -RE selon ses sigles en anglais- ne fonctionne pas d'une manière appropriée. Ce document présente les résultats d'une recherche sur les pratiques de RE. La recherche a été réalisé à travers d'un enquête sur le web et a été dirigé vers les techniques d'élicitation des exigences et vers les techniques de représentation des exigences qui les enquêtés utilisent. Bien que le résultat soit le rapport sur l'état de l'art de la Génie des Exigences pour la population qui a répondu l'enquête, il est probable qu'il reflète l'état de l'art de la RE dans le monde entier.

\footnotetext{
Mots-cles

Génie des exigences, éliciter, techniques d'élicitation, techniques de représentation
} 


\section{INTRODUCCIÓN}

Para los profesionales de la Ingeniería de Software es importante desarrollar nuevas técnicas, métodos, herramientas y notaciones, pero igualmente importante, si no más, es analizar cómo se utilizan en la realidad esas técnicas, métodos, herramientas y notaciones y cuál es el sentimiento acerca de ellas los profesionales en general. Si fuese posible conocer cómo se utilizan en el mundo real, en el futuro será posible diseñar mejores métodos, centrarse en una formación apropiada, automatizar parte de la adopción y evitar los problemas recurrentes que se encuentran al utilizar estos métodos. Con este objetivo, se llevó a cabo una amplia investigación-encuesta para comprender cómo elicitan y cómo especifican los requisitos los ingenieros de software en las diferentes organizaciones.

En la literatura se encuentran varios estudios empíricos acerca de la práctica de la Ingeniería de Requisitos. Emam \& Madhavji [1] realizaron un estudio de campo, acerca de las prácticas de $\mathrm{IR}$ en el desarrollo de sistemas de información, en el que analizaron 60 casos y como resultado descubrieron siete cuestiones técnicas y no-técnicas que influyen en los procesos de IR. Sadraei et al. [2] encuestaron a 16 compañías australianas cerca de la práctica de IR en 28 proyectos de software. Aranda et al [3] investigaron cómo realizan actividades de $\mathrm{IR}$ las pequeñas empresas. El presente trabajo difiere de estos estudios en que se basa en un amplio número de experiencias y observaciones de los ingenieros a través de proyectos. Además, existe un creciente interés por comprender cómo influye la práctica de la Ingeniería de Software globalizada en la IR. Damian \& Zowghi [4] analizaron una organización de desarrollo de software multi-sitio en Australia y determinaron los factores clave que contribuyen al éxito de la gestión de requisitos en la estructura de desarrollo distribuido. Babar \& Verner [5] encuestaron a 12 profesionales de software en Vietnam e investigaron las cuestiones culturales acerca del mantenimiento de la confianza en la tercerización del software. Hickey \& Davis [6] y Sim et al. [7] realizaron encuestas a expertos en requisitos. Estos trabajos se centran en un problema más específico que la comprensión de la situación actual de la industria.

El resto de este documento se estructura así: la sección 2 presenta los métodos de investigación de la encuesta; en la sección 3 se encuentran los datos y los resultados obtenidos; en la sección 4 se detalla la discusión de los resultados en el trabajo y la sección 5 contiene las conclusiones.

\section{MÉTODO DE INVESTIGACIÓN}

Esta investigación se llevó a cabo en 2010 y su objetivo fue investigar el estado de la práctica de IR. Entre las organizaciones que participaron se encuentran empresas estatales, corporaciones multinacionales, empresas privadas nacionales y universidades. La encuesta se estructuró en dos partes principales: 1) se recogieron datos acerca del estado general de los encuestados y sus organizaciones asociadas, para lo que se les pidió responder un cuestionario en línea sobre su práctica regular de IR, en particular, acerca de los enfoques de elicitación de requisitos y las técnicas de representación de requisitos. 2) se recogieron las historias y las observaciones relacionadas con IR. Se esperaba que los resultados de la encuesta reflejaran la práctica industrial y proporcionaran información útil para comprender mejor el estado de la Ingeniería de Requisitos para poder sugerir posibles mejoras en el futuro.

La base de la muestra incluyó a 377 profesionales, procedentes de 237 compañías de software u organizaciones de investigación. Las áreas de negocio de las empresas involucradas cubren diversos segmentos de la industria, como la electrónica y la robótica, entre otros. Todos los participantes en la encuesta son profesionales de software cuyo trabajo diario está íntimamente relacionado con la Ingeniería de Requisitos. En particular, se buscó que los entrevistados narraran, mediante historias, qué han aprendido de sus éxitos y fracasos en IR.

Se contactaron más de 400 personas y se recogieron 377 respuestas, entre las cuales más de 200 personas aportaron historias de éxito o fracaso lo mismo que sus observaciones. Los sujetos encuestados tienen diferentes niveles de experiencia, la mayoría tienen entre 25 y 35 años de experiencia laboral y más del $30 \%$ tienen entre 5 y 10 años. Además, tienen diferentes roles y perspectivas, como altos directivos, gerentes generales y arquitectos de software.

\section{DATOS Y RESULTADOS}

La encuesta se dirigió a las actividades que se realizan en la práctica y que están relacionadas con la Ingeniería de Requisitos.

Pregunta 1: ¿En su organización, que tipo de trabajos se llevan a cabo durante la Ingeniería de Requisitos? Los resultados se muestran en la Figura 1: se puede observar que las actividades de IR que más se practican son la elicitación (65\%), la descripción (59\%), la especificación (67\%) y el modelado y análisis de requisitos (47\%); en comparación, actividades como la Validación de requisitos, el diseño de casos de prueba y la gestión de cambios reciben menos atención.

Pregunta 2: ¿Cuánto esfuerzo se dedica a actividades relacionadas con IR en términos de porcentaje del tiempo del proyecto? En el $42 \%$ de las organizaciones encuestadas, el tiempo dedicado a la IR es más del $10 \%$ de la duración del proyecto, el $25 \%$ dedica más de un $20 \%$ de su tiempo, el $21 \%$ dedica menos del $10 \%$ y el $12 \%$ dedica poco tiempo a esas actividades.

Pregunta 3: "¿Sus clientes están de acuerdo en participar en actividades relacionadas con la Ingeniería de Requisitos?" 120, de los 377 encuestados, afirman que los clientes están contentos de estar involucrados en actividades relacionadas con los requisitos, 185 
respondieron que los clientes son reacios a trabajar en $\mathrm{IR}$, a pesar de que valoran la importancia de los requisitos y de que creen que se debe invertir buen tiempo para poder entregar un documento de alta calidad, los otros 72 encuestados seleccionaron la opción "neutral" como respuesta a esta pregunta.

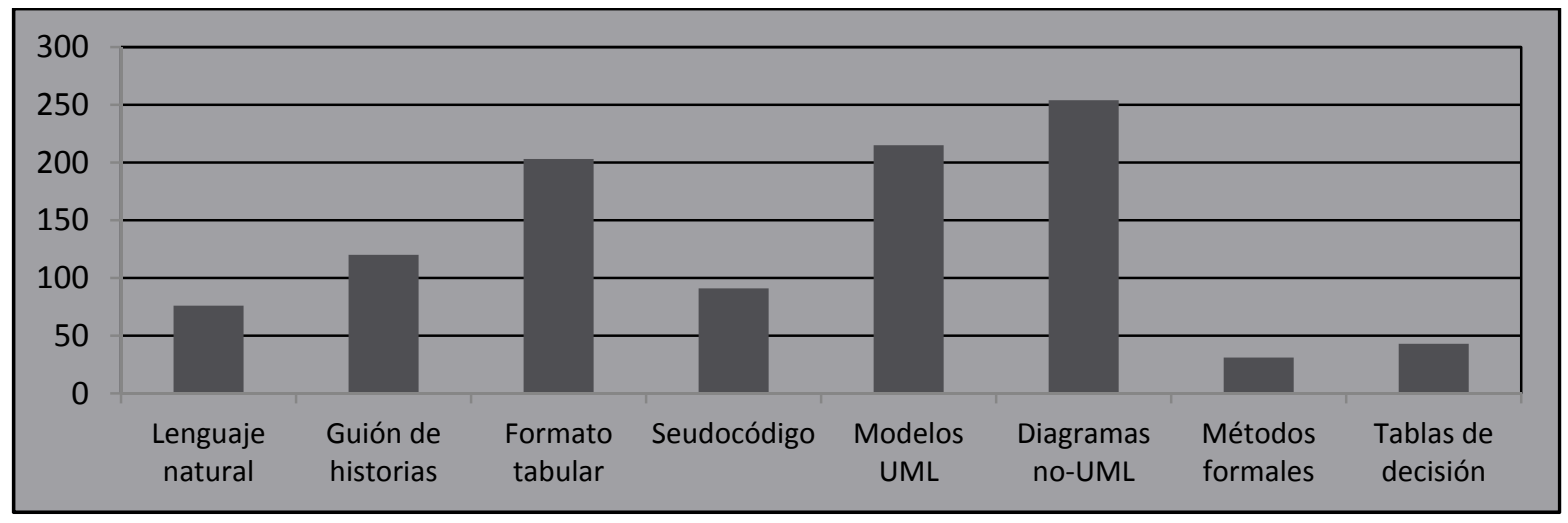

Figura 1. Actividades relacionadas con IR

Pregunta 4: ¿Cree que existe una relación directa entre la especificación de requisitos y la calidad del software? La mayoría de los participantes, 68\%, cree que la calidad del documento de requisitos tiene fuerte impacto en la calidad del producto software final. Sólo el $3 \%$ cree que no hay ninguna relación entre la especificación y la calidad del software y el 29\% restante atribuye un impacto moderado de las especificaciones sobre la calidad del software. Existe una alta correlación entre esta pregunta y la pregunta 2; en general, si las personas piensan que la especificación de requisitos no tiene gran impacto en la calidad del software, no van a invertir tiempo para desarrollarla.

\subsection{Técnicas de elicitación de requisitos}

Pregunta 5: ¿Qué método utiliza para elicitar requisitos? Los resultados del estudio se resumen en la Figura 2. 333 encuestados mencionaron que la entrevista cara a cara es el principal enfoque que utilizan, 206 afirman que siempre requieren de un prototipo mientras elicitan requisitos y 203 siempre se refieren a sistemas similares existentes para elicitar requisitos.

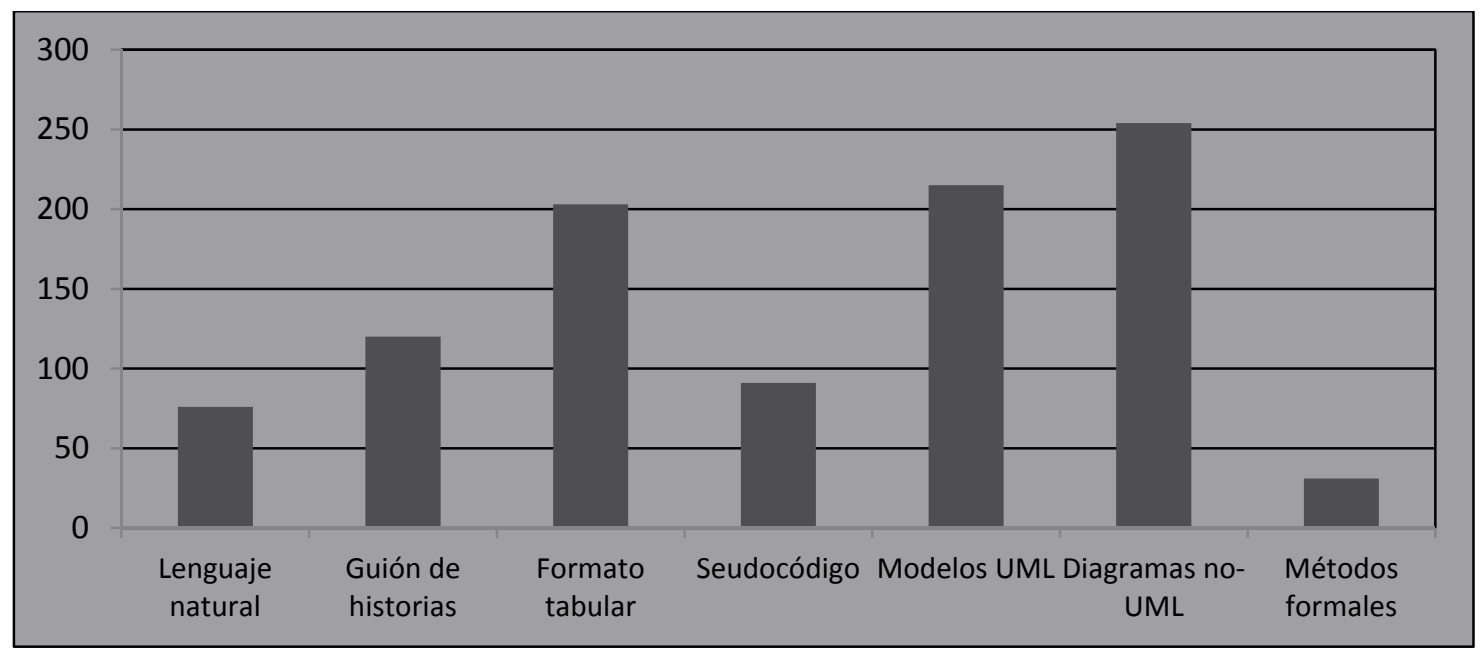

Figura 2. Técnicas preferidas para elicitar requisitos

También se indagó por la actitud de los participantes respecto de los cambios en los requisitos. Pregunta 8: ¿Los clientes hacen cambios en los requisitos incluso después de que el contrato de desarrollo se ha firmado? ¿Cómo enfrenta esta situación? La mayoría de los participantes, el 90\%, piensa que los cambios a los requisitos son normales, el $80 \%$ dice que negocia con los clientes un plan de compromiso, el $7 \%$ realiza lo que el cliente pida y el $14 \%$ simplemente se ciñe al contrato.

\subsection{Técnicas de representación de requisitos}

Pregunta 9: ¿Qué técnicas de representación utiliza? Esta es una pregunta con respuesta de opción múltiple. Los resultados se muestran en la Figura 3. La mayoría de los encuestados seleccionaron la opción seis, es decir, que utilizan diagramas diferentes a los de UML, incluyendo diagramas de flujo de datos o los diagramas ad-hoc que utilizan los ingenieros.

Pregunta 10: ¿Cuáles son las herramientas de IR que utiliza en su práctica diaria? el $40 \%$ informó que utilizan herramientas como DOORS y el resto que no utiliza ninguna herramienta de IR.

Pregunta 11: ¿Cuáles son los contenidos reales que se incluyen en la especificación de requisitos? De acuerdo con los resultados del estudio, los contenidos 
que usualmente se incluyen en el documento de IR son: $78 \%$ descripción y objetivo del proyecto, $99 \%$ descripción de requisitos funcionales, $74 \%$ el esquema general, 64\% descripción de requisitos no funcionales y $50 \%$ registro de cambios. Usualmente, en el documento de requisitos se incluyen los objetivos y el plan del proyecto y la descripción de los requisitos funcionales, mientras que en algunos proyectos se incluye información sobre análisis de riesgos, cambios y agenda, pero comparativamente reciben menos atención.

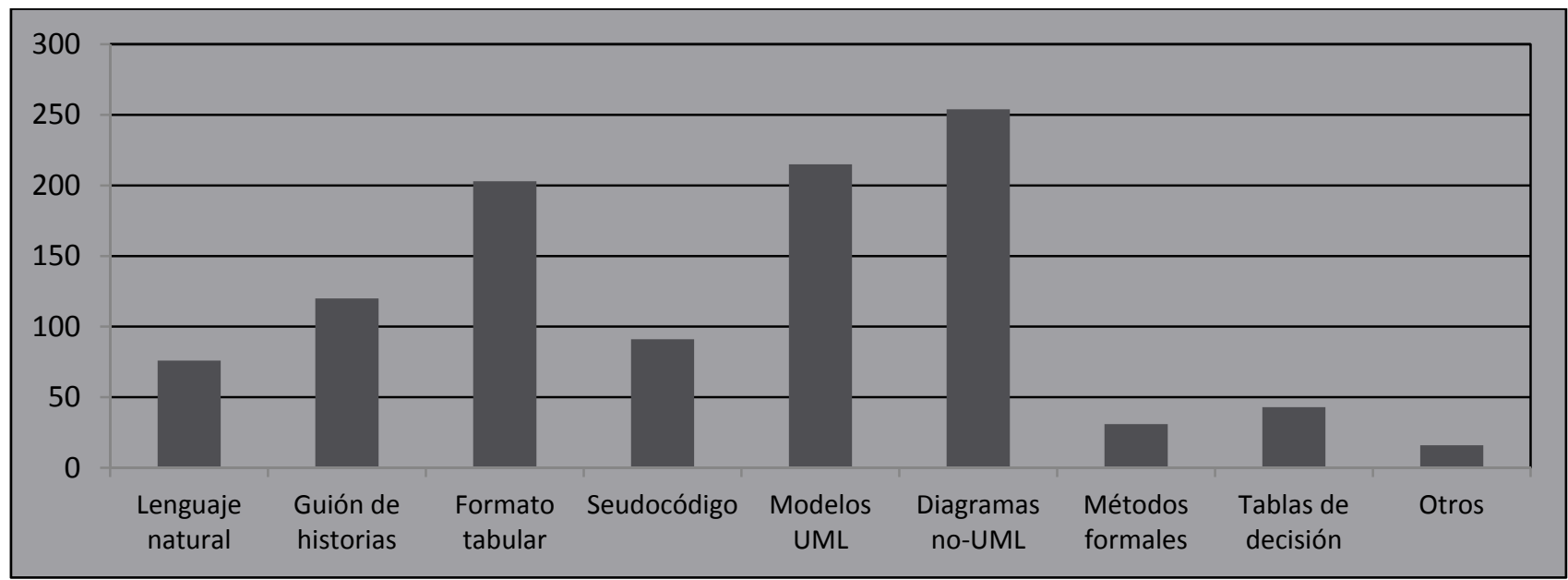

Figure 3. Técnicas de representación de requisitos

\subsection{Fracasos, recomendaciones e historias de éxito}

En el cuestionario, también se pidió a los encuestados que compartieran algunas historias, recomendaciones $\mathrm{u}$ opiniones relacionadas con la práctica de la Ingeniería de Requisitos en sus organizaciones. De los 373 encuestados, 203 proporcionaron información en esta columna. Algunos ejemplos son:

Historia 1: "En uno de nuestros proyectos intercambiamos información con los clientes durante un mes y recogimos abundante información sobre los requisitos, que nos pareció bastante completa en ese momento. El producto se desarrolló en los siguientes tres meses, período durante el cual no fueron necesarios nuevos requisitos o cambios. Sin embargo, cuando estábamos a punto de entregar el producto, tres meses más tarde, el líder rechazó muchas de las funciones anteriormente confirmadas, lo que condujo a otro sistema de procesos de tres meses".

Historia 2: "Uno de nuestros proyectos consistió en desarrollar un sistema de información para gestionar pensiones, la duración del proyecto fue de 9 meses. En la fase de requisitos, no estábamos muy familiarizados con los requisitos del negocio de la gestión pensiones, por lo que el producto entregado en el proyecto sólo incluyó las funcionalidades actuales, que no podían adaptarse a la rápida evolución del negocio en lo que tiene que ver con la gestión de pensiones. Seis meses después, y como consecuencia del cambio de las principales reglas del negocio, hemos perdido una serie de contratos subsiguientes".

Historia 3: "Una vez tuvimos un proyecto para desarrollar el software a una cadena de alquiler de video, en el que el cliente expresó su interés de utilizar el mismo producto para expandirse internacionalmente. Sin embargo, sólo más tarde llegamos a conocer el hecho de que los estándares en el extranjero son muy diferentes a los nacionales, por lo que el producto tuvo que ser completamente reimplementado para cumplir con este requisito".

Al analizar las 203 historias, se encontró que en esta población las prácticas de IR son muy diversas, que dependen del tipo de software que desarrolla la organización, del tipo de producto y del dominio de aplicación particular de ese producto. El 15\% de los encuestados que relataron historias y opiniones trabajan en corporaciones multinacionales, el $17 \%$ están trabajando con empresas patrocinadas por el gobierno ocupando áreas dominantes de la economía nacional, el 53\% laboran con empresas privadas nacionales que crecen rápidamente y que juegan un papel activo en la economía nacional, el 8\% en universidades e institutos de investigación y el $7 \%$ no informó al respecto. En esta investigación se encontró que la cultura y los valores de la organización y el nivel de madurez técnica juegan un papel vital en la IR.

1. Estado de la Ingeniería de Requisitos en las corporaciones multinacionales. Con base en los resultados de la encuesta a 33 organizaciones multinacionales, cuyas sedes se encuentran en los EE.UU., Japón, Suecia, Alemania, Finlandia y la India, el estado de la práctica de la Ingeniería de Requisitos, por lo general, es más avanzada en unos países que en otros, lo mismo que el estado del arte de la tecnología; además, que comprenden más profundamente los principios relacionados con los requisitos y que las herramientas son más accesibles y están disponibles para los profesionales en general.

- La IR es una tarea que se planifica y apropia explícitamente en proyectos de desarrollo de software. 
- Generalmente, cuentan con equipos especiales para manejar los requisitos de los clientes que pueden ser personas de pre-venta 0 de marketing, con un director de proyecto y un proveedor de servicios que participan en la toma de decisiones.

- En la actualidad, las actividades relacionadas con los requisitos se llevan a cabo y se controlan en la sede de la empresa.

- Utilizan múltiples técnicas para la elicitación y la especificación, combinadas en un mismo proyecto; el prototipado se utiliza en muchas empresas para facilitar la comunicación y los modelos UML se utilizan para representar los requisitos y el diseño.

- Utilizan herramientas de gestión de proyectos para controlar y gestionar procesos de requisitos. Las herramientas más utilizadas son DOORS y Team Center.

- La especificación de requisitos detallada se utiliza para comunicarse con el usuario y con el equipo de desarrollo y para obtener su confirmación de forma iterativa.

- Las funcionalidades, las características y los requisitos no funcionales, como rendimiento y seguridad, varían cuando el objetivo se orienta a diferentes mercados.

- Cuando de antemano no hay ningún requisito claro para algunos productos de I $+\mathrm{D}$, el objetivo principal es explorar la factibilidad técnica del diseño.

2. Estado de la IR en las empresas estatales. En las respuestas de los encuestados en 31 empresas estatales del sector energético, productos farmacéuticos, informática y electrónica para el hogar, se determinó que el estado de la práctica de la IR se ve afectado por el nivel de desarrollo de las zonas geográficas donde se ubican.

- No tienen personal dedicado a tareas relacionadas con los requisitos. Es probable que los ingenieros de requisitos no-profesionales utilice una sola técnica para realizar sus actividades, lo cual afecta la calidad final.

- No son consientes de la necesidad de profesionales en IR. El éxito de los proyecto se confía a acciones heroicas en vez de a procesos de gestión estandarizados.

- Hay lagunas evidentes en la comunicación entre el cliente y el equipo de desarrollo. Los usuarios no entienden y no están consientes de los esfuerzos necesarios para implementar determinada funcionalidad. Los diseñadores no siempre prestan suficiente atención a la retroalimentación de los clientes y a menudo definen requisitos en su propio beneficio.
- El nivel de madurez en la práctica de la Ingeniería de Software dentro de la organización tiene gran influencia en la eficiencia de las actividades de los requisitos.

- El nivel de negocio y el conocimiento técnico tienen gran impacto en la calidad de la elicitación de requisitos y en el proceso de gestión. La no-disponibilidad, o la disponibilidad, de un buen conocimiento del dominio contribuye al fracaso, o al éxito, del proyecto. La mezcla de especialistas en el dominio y de especialistas en software también ha demostrado ser útil.

- Hay una jerarquía de poder estricta en este tipo de organizaciones. Por lo tanto, las actividades de requisitos y la toma de decisiones necesitan el apoyo del equipo de alta gestión. En este caso, tiene que planificarse las contramedidas sociales y las políticas de los riesgos del proyecto. En particular, los cambios en la organización del cliente a menudo resultan en cambios radicales, como terminar o rehacer el proyecto de software.

- Las soluciones de diseño maduran sólo después de que un proyecto se ha implementado un par de veces. La personalización basada en una solución de diseño probado es una buena práctica a seguir pero, al mismo tiempo, existen informes sobre proyectos desastrosos provocados por una inadecuada reutilización.

- Es esencial para el éxito del proyecto que los usuarios participen en el diseño conceptual del sistema y en la confirmación del diseño detallado de las funciones del negocio. Sin embargo, en muchos casos, los usuarios rechazan requisitos en las últimas etapas de los proyectos que ya han sido confirmados. Por lo tanto, es necesario predecir de alguna forma los posibles cambios.

- El método de prototipado rápido es adecuado para elicitar requisitos en cualquier tipo de organización. Los prototipos deben evolucionar en función de los cambios en los requisitos y las últimas tecnologías disponibles. Los cuestionarios se consideran menos eficaces.

3. Estado de la IR en las empresas privadas nacionales. Con base en las historias de 32 empresas privadas nacionales en telecomunicaciones y software, se encontró que los ingenieros suelen invertir más esfuerzo en comprender los requisitos involucrando a sus clientes, especialmente cuando se enfrentan a clientes importantes como las agencias gubernamentales. En muchos casos, los requisitos se identifican a partir de múltiples fuentes utilizando varios enfoques. Por otro lado, los clientes usualmente confían menos en las pequeñas empresas privadas nacionales, ya que no están a la 
altura de la gestión y de los procedimientos formales de control de calidad que exigen.

- La documentación sirve principalmente para el propósito de la confirmación al cliente en lugar de referencia técnica. A veces no se pasa al equipo de desarrollo.

- Muchos documentos de especificación de requisitos no cumplen con plantillas estándar. Los requisitos del sistema no se trazan desde la estrategia empresarial, sino más por conveniencia de diseño.

- Los documentos de requisitos usualmente se preparan en MS Word, Excel y Visio y rara vez se utilizan herramientas comerciales. Los ingenieros sienten la urgente necesidad de herramientas de desarrollo rápido de prototipos, como herramientas libres y de código abierto.

- Los ingenieros también sienten la deficiencia de un dominio estandarizado de la base de conocimientos de los requisitos, o por lo menos directrices para enmarcar el diseño en cada dominio de aplicación reconocida.

- A menudo, la priorización de requisitos se relaciona con la disponibilidad de mano de obra de desarrollo y su área de experiencia.

- A veces, el éxito del proyecto se apoya en los logros heroicos de los ingenieros y en las experiencias individuales de los gestores de proyectos, en lugar de técnicas y de procesos demostrados en la mayoría de las empresas. La elicitación de requisitos no es meramente técnica, a menudo es social y política. A veces el éxito de los proyectos se ve obstaculizado por posibles conflictos de intereses y conductas evasivas. Los requisitos de toma de decisiones pueden ser intuitivos y prematuros.

- Cuando se elicitan requisitos de agencias del gobierno o servidores públicos, las personas no están muy abiertas a discutir sus propias posiciones y opiniones y suelen ser pasivas y conservadoras. Por lo tanto, es importante organizar una reunión de grupo con personas de rango similar, para que las discusiones sean más vivas y libres.

- Al llamar a una reunión de grupo para elicitar requisitos, un escenario típico es que sólo una 0 dos personas participen y que la mayoría guarde silencio o hable poco. Pero, cuando se presenta el documento de requisitos, a menudo le hacen gran cantidad de modificaciones.

\subsection{Estado de la IR de acuerdo con el tipo de producto}

También, con base en el análisis de estas historias, se encontró que la selección de los métodos de Ingeniería de Requisitos depende de los clientes destino. De las respuestas recogidas en la encuesta, el 10\% proviene de empresas orientadas a la contratación externa de productos, el $16 \%$ de empresas orientadas a la comercialización masiva de productos y el $74 \%$ de empresas dedicadas a desarrollar productos a la medida. Para elicitar los requisitos del software de comercialización masiva se requieren los métodos de investigación de mercado, mientras que las técnicas de relaciones humanas son más importantes para productos a la medida. Cuando se trata de desarrollo de software por outsourcing se requiere la investigación transcultural, especialmente en culturas complejas, como en Japón o China.

1. Productos software de comercialización masiva. La elicitación de requisitos para productos de consumo masivo se considera difícil. El siguiente es un resumen de las experiencias compartidas por los encuestados en sus historias.

- Identificar potenciales grupos de usuarios representativos es un paso esencial para la elicitación de los requisitos de este tipo de software.

- Los cuestionario se utilizan con más frecuencia en la elicitación de requisitos; la calidad de las respuestas depende en gran medida de los incentivos a los participantes $y$ de la capacitación a los clientes.

- En algunos casos se envió un Email con un cuestionario adjunto, pero el resultado fue miserable. Los datos devueltos no se pueden utilizar para el análisis.

- El método de encuesta es relativamente útil para entrevistar a los clientes cara a cara después de un determinado programa de formación.

- Cuando los clientes están más familiarizados con las funcionalidades del software, se pueden invitar a que respondan un cuestionario. Por lo tanto, los datos recogidos son de calidad relativamente alta.

- El prototipo siempre será un medio útil para recoger las reacciones de los usuarios. Cotidianamente, a los analistas de requisitos se les paga menos que a los desarrolladores, por lo que en muchos casos no tienen suficiente conocimiento y experiencia como para entregar una especificación de requisitos de alta calidad.

- La elicitación de requisitos de estos productos puede ser embarazosa cuando se confrontan competidores y clientes defensivos.

2. Productos software por contratación externa. Muchos requisitos de proyectos outsourcing son vagos $y$ les falta información detallada. A continuación un resumen de las experiencias compartidas por los encuestados en sus historias. 
- Existen grandes brechas de comunicación entre quien desarrolla y quien contrata.

- A menudo, la elicitación de requisitos se hace en el país del outsourcing y los ingenieros lo consideran como un problema para desarrollar ciertas características con base en suposiciones y "pensamiento libre".

- Los proyectos outsourcing están en mejores condiciones; el modo habitual de trabajo para la deslocalización es que el desarrollo del proyecto y la elicitación de requisitos se llevan a cabo en la oficina del outsourcing y el desarrollo y el proceso de pruebas es más controlado y más rígido.

- El equipo de desarrollo debe estar consciente de situaciones en las que la diferencia en el trabajo, la vida y el pensamiento llevan a diferentes requisitos del sistema en desarrollo y que no deben adicionar detalles incompatibles con las funciones originales requeridas.

- Los procesos de requisitos para proyectos internacionales están muy influenciados por el idioma, las diferencias geográficas y culturales y por barreras como monedas múltiples, idiomas múltiples, etc.

- Se debe ejecutar una mayor iteración de comunicación y confirmación. El lenguaje es una cuestión fundamental, por ejemplo, en algunos idiomas existen expresiones que se diferencian explícitamente y eso se debe tener en cuenta.

- Además, las personas procedentes de diferentes países a menudo tienen diferentes preferencias sobre requisitos no funcionales. Unos clientes prefieren la facilidad de uso y una interfaz de usuario elegante, mientras que otros prefieren la estabilidad y la fiabilidad.

- Incluso, para personas que hablan un mismo idioma, todavía se siente la imperiosa necesidad de la comunicación cara a cara para fines cognitivos.

- En algunos casos, se prefieren las videoconferencias a las teleconferencias para lograr un entendimiento común compartido.

\section{CONCLUSIONES}

Todo lo anterior es interesante para comprender el estado actual de las prácticas industriales en mercados de rápido crecimiento, lo mismo que para todo el mundo. En la primera fase de esta investigación se recogieron y analizaron las experiencias y las historias relacionadas con requisitos, en proyectos reales y en varios dominios de la industria. Los resultados mostraron que el estado de la práctica de la Ingeniería de Requisitos varía de una organización a otra. Además, que el nivel de conocimientos técnicos y la experiencia en el campo determinan la eficiencia de las actividades de IR. En resumen, las razones más importantes por las cuales falla la práctica de la Ingeniería de Requisitos son:

- Los clientes no tienen una comprensión clara de los requisitos del sistema, incluyendo el alcance del mismo, las principales características funcionales y los atributos no-funcionales.

- Las necesidades y la comprensión de los usuarios cambia constantemente.

- Los ingenieros de software no tienen suficiente acceso al conocimiento y a la experiencia del dominio.

- El cronograma del proyecto es demasiado estrecho como para permitir una adecuada interacción y un periodo de aprendizaje entre el cliente y el equipo de desarrollo.

- La reutilización de diseños existentes tiene un contexto y un entorno equivocados.

- A los tomadores de decisiones en requisitos les falta conocimientos técnicos y del dominio.

- Faltan vínculos de comunicación entre clientes, analistas y desarrolladores.

- Falta un dominio estandarizado de definición de datos y de interfaz del sistema y del entorno.

Con base en los resultados, también se sugieren las siguientes mejoras a las prácticas actuales de Ingeniería de Requisitos:

- Mejorar el proceso de gestión de proyectos para facilitar la comunicación, la documentación y el control y de gestión de cambios.

- El conocimiento del dominio y los prototipos son condiciones necesarias para el éxito de la práctica de la IR.

- Hacer que el cliente sienta que los requisitos y el sistema futuro son de su propiedad y por lo tanto su responsabilidad.

- Ser proactivo en los proceso de IR y ser capaz de predecir posibles cambios y necesidades futuras.

- Enlazar los requisitos con las pruebas y adoptar un proceso de diseño basado en pruebas.

- Aun cuando el tiempo es escaso, debe ser claro qué desarrollar antes de cómo desarrollarlo.

- Desarrollar herramientas de IR que se ajusten mejor a las necesidades reales de los clientes y de los ingenieros.

Para este proyecto se optó por encuestas basadas en la web, pero se podrían haber elegido otros métodos diferentes de investigación para el estudio adicional. A pesar de que es simple y efectivo, el método basado en encuestas no puede reflejar todo lo que los sujetos encuestados tienen en mente. Por lo tanto, para otros estudios de seguimiento se pueden elegir otros métodos de investigación, como la entrevista cara a cara y el taller, para explorar a fondo los puntos de vista reales de los sujetos. Aunque este estudio estuvo limitado por su alcance y su escala, se espera que los resultados sean útiles para los profesionales de la industria y para los investigadores académicos que deseen mejorar las prácticas actuales. 


\section{REFERENCIAS}

[1] K. El Emam \& N. H. Madhavji. "A Field Study of Requirements Engineering Practices in Information Systems Development". Proceedings of the Second IEEE International Symposium on Requirements Engineering. York, England, Vol. 0, pp. 68-80, 1995.

[2] E. Sadraei et al. "A field study of the requirements engineering practice in Australian software industry". Requirements Engineering Journal, Vol. 12, No. 3, pp.145-162, 2007.

[3] J. Aranda, S. Easterbrook \& G. Wilson. "Requirements in the Wild: How Small Companies Do It". Proceedings of the 15th IEEE International Conference on Requirements Engineering (RE'07). New Delhi, India, pp. 39-48, 2007.

[4] D. E. Damian \& D. Zowghi. "The Impact of Stakeholders? Geographical Distribution on Managing Requirements in a Multi-Site Organization". Proceedings IEEE Joint
International Conference on Requirements Engineering (RE'02). Essen, Germany, pp.319-330, 2002.

[5] M. Ali Babar, J. M. Verner \& P. T. Nguyen. "Establishing and maintaining trust in software outsourcing relationships: an empirical investigation". Journal of Systems and Software, Vol. 80, No. 9, pp.1438-1449, 2007.

[6] A. Hickey \& A. Davis. "Elicitation Technique Selection: How Do Experts Do It?" Proceedings of the 11th IEEE International Conference on Requirements Engineering (RE'03), Monterey, USA, pp. 169-178, 2003.

[7] S. E. Sim, T. A. Alspaugh, \& B. Al-Ani. "Marginal Notes on Amethodical Requirements Engineering: What experts learned from experience?" Proceedings of the 16th IEEE International Requirements Engineering Conference (RE'08), Barcelona, Spain, pp. 105-114, 2008. 